\title{
Impactos da COVID-19 no setor cultural português: primeiros apontamentos de um estudo em curso
}

\author{
https://doi.org/10.21814/uminho.ed.25.9
}

\section{Manuel Gama}

Manuel Gama (ORCID: 0000-0002-5950-1956) é investigador do Centro de Estudos de Comunicação e Sociedade (CECS) da Universidade do Minho. Áreas de interesse de investigação: Políticas Culturais, Gestão Cultural, Redes Culturais, Mediação Cultural, Públicos da Cultura e Educação Artística. É, desde 2016, um dos coordenadores do Observatório de Políticas de Comunicação e Cultura (POLObs) do CECS-UM. 


\section{NOTA INTRODUTÓRIA}

Seis meses depois de a Organização Mundial da Saúde ter revelado a existência de casos de pneumonia de causa desconhecida em Wuhan (2020) prever os impactos deste acontecimento de saúde pública continua a não ser uma tarefa fácil.

Evoluímos de um vírus desconhecido com uma probabilidade de propagação humana baixa ou muito baixa (Centro Europeu de Prevenção e Controlo de Doenças, 2020), para uma pandemia de um novo coronavírus 2019-nCoV (COVID-19), que, no final de junho de 2020, já tinha contaminado mais de dez milhões de pessoas de 216 países, provocando mais de meio milhão de vítimas mortais.

Para fazer face à pandemia, entidades nacionais e internacionais, nomeadamente da área da saúde pública, foram tomando um conjunto de medidas, que, revelando algumas hesitaçốes e contradiçôes, concorreram de forma decisiva para o evoluir da pandemia e para os impactos que a mesma está a ter um pouco por todo o mundo.

Independentemente da escala de impactos, não restam dúvidas de que nos encontramos num processo de transformação único, que está a ter implicações substantivas em todas as dimensões na nossa vida individual e coletiva. Por isso há que aproveitar a oportunidade e, como diz Latour, no futuro, "a última coisa a fazer seria voltar a fazer tudo o que fizemos antes” (2020).

No que concerne ao setor cultural, tornou-se óbvio desde muito cedo que a imunidade à pandemia não estava assegurada. Sabine Verheye, presidente da Comissão para a Cultura e a Educação do Parlamento Europeu, alertou, no final de março, que os setores culturais e criativos estavam a ser devastados pelo impacto de medidas rigorosas de saúde pública (Parlamento Europeu, 2020). E na reunião de Ministros da Cultura promovida pela UNESCO em abril, assinalou-se, por um lado, a importância fundamental da cultura para fazer face à crise e, por outro lado, o efeito cascata que a crise no setor cultural estava a provocar em outros setores da atividade económica (UNESCO; 2020).

Nesta relação COVID-19 e cultura convocamos simbolicamente Wuhan, cidade epicentro da pandemia. Wuhan aprovou, em fevereiro de 2012, um plano cultural a médio prazo com o objetivo de implementar um conjunto de políticas para promover 
o desenvolvimento cultural inovador, coordenado, aberto e partilhado (Xuanxuan, 2016). Assim sendo, considera-se expectável que o Governo tenha incluído no lote de constrangimentos provocados pela COVID-19 algumas dimensões do plano cultural, que deveriam estar concluídas no ano de 2020 e que dificilmente serão atingidas. Sublinha-se que, para além do simbolismo, o exemplo de Wuhan foi convocado, pelo facto da cidade ter um plano estratégico para a cultura - instrumento de gestão fundamental para o desenvolvimento de políticas culturais coerentes, consistentes, concertadas, continuadas e consequentes, que ainda não é uma prática ancorada a nível mundial -, que foi objeto de avaliação em 2016 e foi considerado, pela comissão de cultura da organização Cidades e Governos Locais Unidos, como uma boa prática no que concerne à relação entre a cultura e o desenvolvimento sustentável.

Saindo de Wuham, mas mantendo o foco nos impactos da COVID-19 no setor cultural, assinala-se a importância da multiplicidade de análises que se iniciaram em março de 2020: à escala regional (e.g. Secretaria da Cultura do Estado do Ceará, 2020), à escala nacional (e.g. Ministério da Cultura do Perú, 2020) e à escala internacional (e.g. European Creative Business Network, 2020), centrados em distintos domínios culturais (e.g. Network of European Museum Organisations, 2020) e desenvolvidas por entidades representativas de setores culturais específicos (e.g La Red Española de Teatros, Auditorios, Circuitos y Festivales de Titularidad Pública, 2020) ou da academia (e.g. Observatório da Economia Criativa da Bahia da Universidade Federal do Recôncavo da Bahia, 2020).

No que concerne a Portugal, à medida que a COVID-19 se foi aproximando de território e do setor cultural português - o que pode ser simbolicamente assinalado, pela notícia do teste positivo ao escritor chileno Luís Sepúlveda à COVID-19, depois de participar no Correntes de Escrita (Correia, A. 2020) e pela confirmação do primeiro caso positivo diagnosticado em Portugal (Maia, 2020), tornou ainda mais nítido que o país e o setor cultural nacional não estavam imunes à pandemia.

Este foi o pano de fundo que concorreu para que o POLObs tenha considerado relevante e pertinente desenvolver um projeto de investigação para identificar e analisar alguns dos impactos da COVID-19 no setor cultural português. 
Assim, "Impactos da COVID-19 no setor cultural português" encontra-se inscrito nas múltiplas análises que se iniciaram em março de 2020, sendo que algumas das suas caraterísticas tornam o projeto distintivo:

1) a operacionalização da dimensão cultural é efetuada através dos dez domínios da cultura definidos em 2016 no âmbito da Conta Satélite da Cultura 20102012 (Instituto Nacional de Estatística I.P., 2016); 2) a análise efetuada é longitudinal, com três janelas temporais (março de 2020, 2o trimestre de 2020 e 2 oㅡㄴ semestre de 2020);

3) a análise é multidimensional, com cruzamento de impactos de natureza variada.

Genericamente, o projeto, que vai decorrer até março de 2021, visa atingir os seguintes objetivos específicos:

- aferir o impacto mediático das consequências da COVID-19 no setor cultural português;

- identificar o fluxo de notícias produzidas pelos municípios e pelas entidades intermunicipais que abordam sincronicamente aspetos relacionados com COVID-19 e cultura;

- analisar as iniciativas do Governo, através do Ministério da Cultura, do Parlamento e de organismos/entidades tutelados pelo Ministério da Cultura, para enfrentar os constrangimentos provocados pela COVID-19;

- avaliar os impactos, esperados e observados, que a COVID-19 teve e terá nas organizaçóes e profissionais do setor cultural português.

No presente artigo, por se tratar de um projeto em curso e de uma apresentação preliminar de resultados, vamos centrar-nos nos dados recolhidos até 31 de maio de 2020 em duas das dimensões do estudo: o impacto mediático das consequências da COVID-19 no setor cultural português e nos impactos, esperados e observados, que a COVID-19 teve e terá nas organizações e profissionais do setor cultural português. 


\section{IMPACTO MEDIÁTICO DAS CONSEQUENCIAS DA COVID-19 NA CULTURA}

Para aferir o impacto mediático das consequências da COVID-19 no setor cultural português, a equipa do projeto optou por fazer um levantamento de notícias nos motores de pesquisa de um grupo restrito de websites de jornais e revistas portuguesas selecionadas, nomeadamente, a partir dos valores referentes à circulação paga (impressa e digital) do quarto trimestre de 2019 disponibilizados no website da Associação Portuguesa para o Controlo de Tiragem e Circulação. Aos sete jornais e revistas que constituem o grupo, juntou-se a Lusa - Agência de Notícias S.A. (Lusa), por nos permitir chegar a um leque mais abrangente de notícias. Para a identificação das notícias utilizaram-se três palavras-chave (COVID-19, coronavírus e cultura) - utilizadas isoladamente ou associando as duas primeiras à terceira. De cada notícia identificada, registaram-se um conjunto de dados para nos permitir efetuar, primeiro uma análise de natureza quantitativa e, posteriormente, uma análise de natureza qualitativa em função de um conjunto de categorias definidas para o efeito.

Assim, o processo de aferição do impacto mediático das consequências da COVID-19 no setor cultural português inclui o levantamento de notícias nos oito websites selecionados. Os itens identificados são fragmentados em função do website de origem e da dimensão temporal. Posteriormente é efetuada uma análise aos títulos das notícias a partir das palavras mais usadas e por último a análise de conteúdo de um conjunto específico de notícias. Esta dimensão do projeto integra ainda a análise das primeiras páginas dos sete jornais e revistas objeto do estudo.

A pesquisa de notícias permitiu a identificação de um total de 533 itens no mês de março e de mais 1.492 noticias nos dois primeiros meses do segundo trimestre de 2020, perfazendo 1.975 itens publicados entre 1 de março e 31 de maio.

Uma primeira análise quantitativa da amostra revela que a maioria das notícias são provenientes da Lusa (26,1\%), sendo o Público, o Jornal de Notícias e a Visão, responsáveis, respetivamente, por $18,2 \%, 13,1 \%$ e $12,6 \%$ das notícias identificadas.

Não obstante ter sido o mês com menos publicações identificadas (26,5\%), não deixa de ser relevante convocar algumas das notícias publicadas durante o mês de 
março, tanto mais que nos permitem perceber que os tempos de reação por parte de algumas entidades com responsabilidades no setor cultural foram muito distintos. Das 182 notícias publicadas na primeira quinzena, destacamos a que refere a iniciativa da autarquia da Porto de reunir "com representantes de empresas privadas da área da cultura e do turismo "para alinhar estratégias" relativamente à pandemia" (Cardoso, 2020), a que dá conta do encerramento dos museus, monumentos e palácios nacionais afetos à Direção-Geral do Património Cultural (DGPC) e dos museus e monumentos dependentes das direçóes regionais de Cultura do Norte, Centro, Alentejo e Algarve (Lusa, 2020a) e, por fim, a que sublinha as preocupaçóes do Sindicato dos Trabalhadores de Espetáculos, do Audiovisual e dos Músicos (CENA-STE) no que concerne aos "direitos dos trabalhadores da área da Cultura, bem como das estruturas que cancelem ou adiem iniciativas seguindo as recomendações vigentes” (Lusa, 2020b). Foi necessário esperar por mais de duas centenas e meia de notícias identificadas nos oito websites consultados para, às $17 \mathrm{~h} 32$ do dia 19 de março, a Lusa publicar a notícia da criação, por parte do Ministério da Cultura, de um endereço de correio eletrónico para “dar resposta às dúvidas do setor sobre todas as medidas de apoio" face à COVID-19 (Lusa, 2020c), sendo que no dia 20 março surgiram mais informaçôes sobre as medidas de apoio do Ministério da Cultura a artistas e profissionais do setor (Lopes, 2020), que foram sendo aprofundadas ao longo da quinzena (e.g. Cabrita, 2020; Cardoso, J. 2020; Público, 2020a). Dos 351 itens que foi possível identificar na segunda quinzena de março, vamos completar a lista de destaques com a notícia que nos apresenta um conjunto de argumentos de como pode a cultura ajudar nesta época de pandemia (Martins, 2020), com as notícias sobre as preocupações de organizaçốes representativas do setor - como a Sociedade Portuguesa de Autores (Lusa, 2020d) e a Plateia (Lusa, 2020e) -, com as notícias com linhas de financiamento criadas por entidades não dependentes do Ministério da Cultura - como a Fundação Calouste Gulbenkian (Correia, J. 2020) e o município de Lisboa (Carrapatoso, 2020) - e com as notícias referentes a estudos para avaliar os impactos da COVID-19 no setor cultural - como o do CENA-STE (Público, 2020b) e o da Fundação Gestão dos Direitos dos Artistas (Lusa, 2020f).

O número de notícias identificadas no mês de abril foi ligeiramente superior ao identificado no mês de maio, mas a realidade é que, nos três primeiros meses do estudo, 
o pico de notícias aconteceu na segunda quinzena de maio, representando $21,7 \%$ da totalidade de notícias publicadas entre março e maio sobre a temática COVID-19 e cultura. A análise dos títulos das notícias identificadas permite perceber que o elevado volume de notícias na segunda quinzena de maio pode ter múltiplas justificações, como: a abertura de equipamentos culturais, nomeadamente "os novos desafios dos museus: menos visitantes e menos receita” (Caetano, 2020); o reagendamento de eventos culturais “em virtude da proibição de festivais de música até 30 de setembro" (Costa, 2020) e as polémicas em torno das linhas de financiamento criadas pelo Governo (Soldano \& Nadais, 2020).

A análise das palavras mais usadas nos 1.975 títulos das notícias, também nos está a permitir identificar algumas das ideias-chave que atravessam a amostra:

1) impactos negativos (e.g. adiamento/cancelamento de eventos, suspensão de atividades, encerramento de instalaçôes);

2) papel do Governo e das autarquias locais na mitigação dos impactos;

3) iniciativas concretas promovidas por profissionais e organizaçôes do setor cultural.

No que concerne às 447 capas de jornais e revistas analisadas entre março e maio, verificou-se que o peso da cultura nas 4.112 chamadas de primeira página é residual: $1,8 \%$ do total das chamadas abordam sincronicamente aspetos relacionados com cultura e a COVID-19, aos quais se acrescem 2,5\% das chamadas de capa dedicadas exclusivamente à cultura.

De entre as primeiras páginas consultadas, foi no Jornal de Notícias do dia 2 de março que foi possível identificar a primeira chamada com a temática da COVID-19 e cultura, que se centrava no facto de a "Póvoa de Varzim [estar] em alerta com coronavírus de Luís Sepúlveda” (Jornal de Notícias, 2020a), mas foi no Público que se encontraram mais primeiras páginas dedicadas, quase em exclusivo, aos impactos da COVID-19 no setor cultural - as capas de 20 de março (Público, 2020c) e de 22 de maio (Público, 2020d). No que diz respeito às temáticas, a grande maioria das chamadas de primeira página sobre COVID-19 e cultura, centrou-se inicialmente nos impactos negativos (e.g. adiamento/cancelamento de eventos, suspensão de 
atividades, encerramento de instalaçóes, constrangimentos financeiros), para, mais tarde, abordar de forma menos veemente as reivindicações dos profissionais do setor e os processos de reabertura dos espaços culturais. Não deixa de ser interessante sublinhar que as chamadas de primeira página sobre medidas e iniciativas da tutela para mitigar os impactos da COVID-19 foram, praticamente, nulas - a título meramente ilustrativo, refere-se que a palavra ministra só figura por duas vezes nas primeiras páginas analisadas, sendo que uma das vezes foi para noticiar que os "artistas doam 151 euros à ministra” (Jornal de Notícias, 2020a).

\section{IMPACTOS NOS PROFISSIONAIS E ORGANIZAÇÕES DO SETOR CULTURAL}

Para avaliar os impactos, esperados e observados, que a COVID-19 teve e terá nos profissionais e organizaçóes do setor cultural português, decidimos aplicar um inquérito por questionário, que se manterá ativo até 31 de dezembro de 2020.

$\mathrm{Na}$ construção do inquérito seguiram-se os procedimentos metodológicos habituais no desenho de instrumentos desta natureza e na sua aplicação foram seguidos todos os princípios éticos, nomeadamente no que concerne à confidencialidade, ao consentimento informado e à possibilidade de recusa na participação. Tendo em consideração os objetivos gerais do estudo, optou-se por construir duas versões do questionário, uma destinada a profissionais e outra a organizaçôes do setor cultural. A estrutura base das duas versões do questionário é semelhante e integra duas secçôes principais: 1) caracterização do respondente e 2) avaliação dos impactos da COVID-19 na atividade. Com a secção de caraterização dos profissionais e das organizações do setor cultural, pretendeu reunir-se um conjunto de informaçôes sobre os respondentes (e.g. domicílio fiscal, ano de início de atividade profissional regular e continuada, vínculo profissional/natureza jurídica, número de postos de trabalho no caso das organizaçóes culturais, domínios e funções culturais da atividade profissional, volume de negócios no ano de 2019). Com a secção de avaliação dos impactos da COVID-19 procurou-se reunir informaçôes sobre várias dimensões (e.g. atividade profissional, volume de negócios, recursos humanos das organizações culturais) e em três tempos distintos (até 31 de março, de 1 de abril a 30 de junho, de 1 de julho a 31 de dezembro). Na segunda 
seção das duas versões do questionário foi ainda solicitado que fosse indicada aquela que poderá ser a mais relevante consequência que a COVID-19 poderá provocar, em 2020, no tecido cultural português. Composto essencialmente por perguntas fechadas, as duas versões do questionário foram alojadas na plataforma LimeSurvey, sendo que o convite à participação foi efetuado, a partir de 20 de março, através dos canais de divulgação habituais do POLObs, mas também por entidades, públicas e privadas, do setor cultural português.

Segue-se a apresentação sumária dos resultados referentes aos primeiros dois meses em que o questionário esteve ativo - de 20 de março a 19 de maio de 2020.

Dos 799 profissionais e das 382 organizações do setor cultural português que se registaram na plataforma para preencher o questionário, 33,5\% submeteram o questionário. Depois de concluído o processo de validação, 3,8\% dos questionários foram considerados inválidos, concorrendo para que a amostra atual do estudo seja constituída por 271 profissionais e 110 organizações do setor cultural, que preencheram ou atualizaram o questionário.

Os dados de caraterização da amostra revelam que a maioria dos respondentes são profissionais do setor cultural, com domicílio fiscal na Área Metropolitana de Lisboa e na região Norte, que iniciaram a sua atividade profissional, regular e continuada, a partir do ano 2000, no domínio das artes do espetáculo e com a função de criação. Realça-se que os dados referentes às organizações estão, à sua escala, alinhados com os dos profissionais e que é possível encontrar na amostra profissionais e organizações de todas as NUTS 2, o que é significativo do alcance do estudo. No que concerne à natureza jurídica das organizações do setor cultural, destaca-se que a maioria dos respondentes são associações, havendo, contudo, cerca de 25,5\% que selecionaram a opção de resposta outro por serem entidades públicas (e.g. bibliotecas, museus, municípios). A análise da questão referente aos recursos humanos, permite perceber que as organizações têm um total de 1.417 trabalhadores, sendo que destes há 32,7\% que são trabalhadores independentes. A grande maioria das organizações tem menos de 10 trabalhadores em cada subcategoria, sendo que uma análise mais fina dos dados revela que tem menos de 10 trabalhadores, independentemente do regime de contratação. Os dados referentes ao vínculo laboral dos profissionais do setor cultural, tornam nítida a precariedade do 
setor, com 63,5\% dos respondentes a serem trabalhadores independentes. Na secção de caracterização dos inquiridos, também se solicitaram informações referentes ao volume de negócios no ano e 2019 e as respostas reforçam, mais uma vez, a fragilidade do tecido cultural português, de que o volume de negócios anual é apenas um indicador: 66,4\% das organizações tem uma média mensal do volume de negócios inferior a $5.000 €$ e o valor mediano do volume de negócios mensal dos profissionais do setor cultural não ultrapassa os $683,54 €$.

Convocados os dados referentes à caraterização da amostra, seguem-se os impactos da COVID-19 no setor cultural português identificados pelos respondentes.

A comparação entre o Gráfico 1 e o Gráfico 2 permite perceber que o cancelamento, efetivo ou esperado, da totalidade ou de até $50 \%$ das atividades previstas é um dos fatores mais preocupantes, nomeadamente no que se refere aos profissionais do setor cultural e no segundo semestre de 2020. Os dados do reagendamento das atividades que foram adiadas devido à COVID-19 evidenciam mais um problema, com 22,4\% das organizações e $29,1 \%$ dos profissionais a afirmarem que ainda não tinham sido definidas as datas para as atividades que se iriam realizar no segundo trimestre, sendo que no que se refere às atividades do segundo semestre, as organizações do setor cultural apresentaram valores previsíveis de adiamentos sem data definida ainda mais elevados (34,3\%).

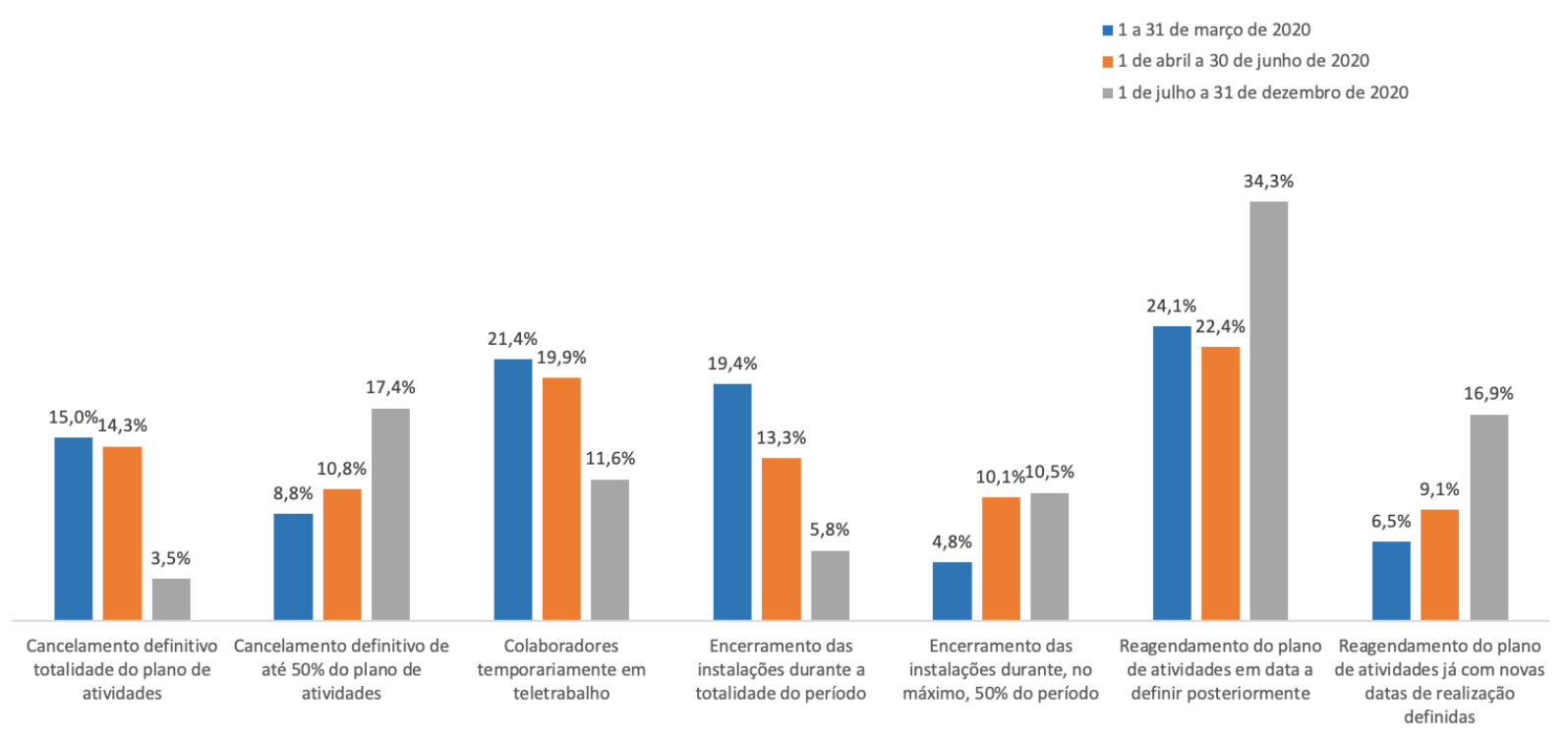

Gráfico 1. Impactos esperados no plano de atividades das organizações do setor cultural. 


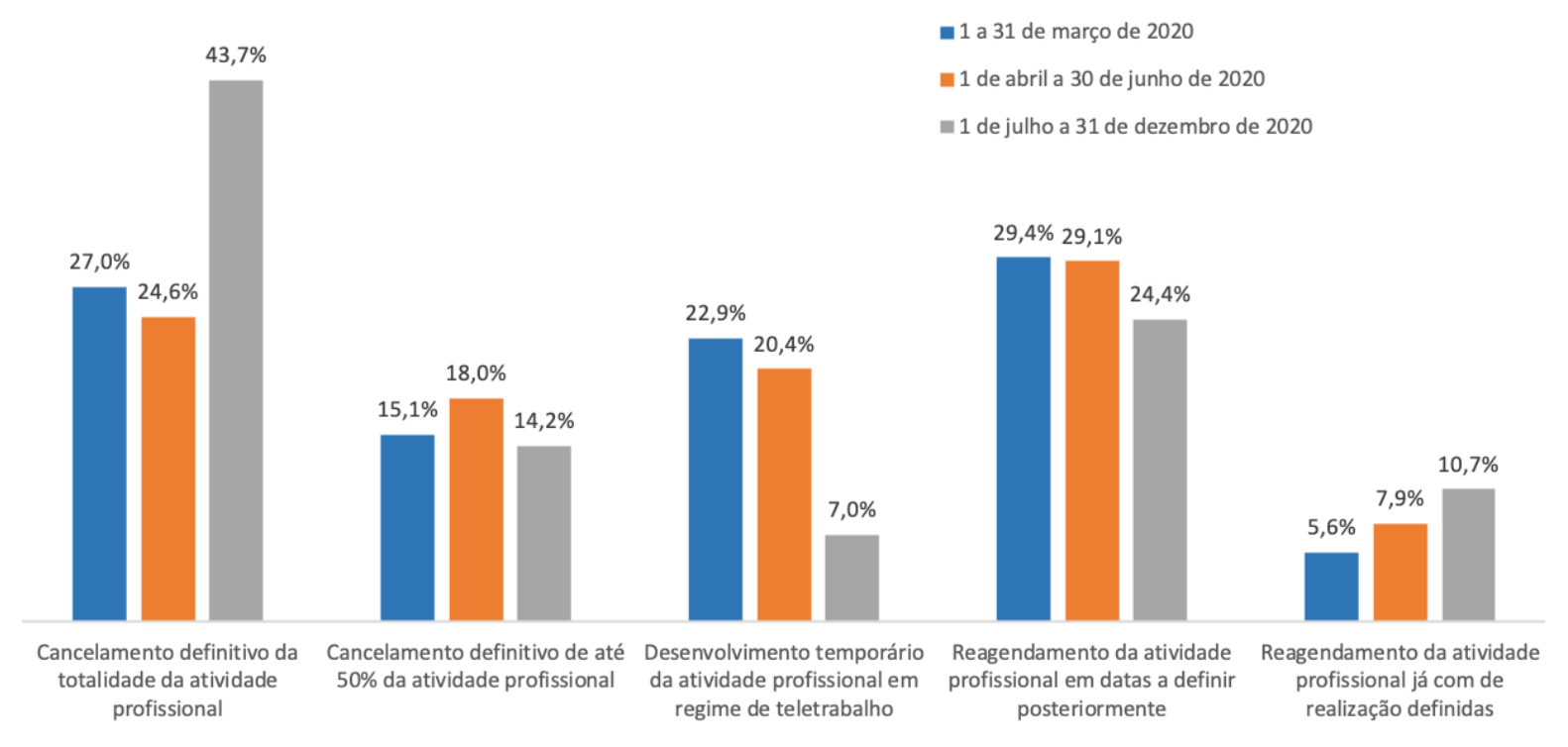

Gráfico 2. Impactos esperados nas atividades dos profissionais do setor cultural.

Os impactos apresentados nos planos de atividades têm, como seria de esperar, reflexo nos recursos humanos das organizações e no volume de negócios das organizações e dos profissionais.

No que se refere aos recursos humanos das organizaçóes, salienta-se que 34,6\% dos respondentes assinalam a redução de postos de trabalho, sendo que 14,6\% afirmam mesmo que terão uma redução superior a 50\%. Se entrarmos em linha de conta que a maioria das organizaçóes têm menos de 10 trabalhadores e que a maioria dos trabalhadores são trabalhadores independentes, podemos estar perante um cenário de uma subida muito significativa da taxa de desemprego no setor cultural e da extinção de um conjunto muito significativo de organizaçóes do setor cultural português. A redução no volume de negócios anual também parece ser um dos impactos esperados, com $48,2 \%$ das organizações e $70,5 \%$ dos profissionais a esperarem uma redução no volume de negócios de, no mínimo, 50\%.

Os dados que acabamos de convocar permitem perceber, de forma muito rápida e objetiva, que os impactos da COVID-19 no setor cultural português serão muitíssimo significativos. 
Mas os contributos dos respondentes ao inquérito não se ficaram pelos dados objetivos. As duas versões do inquérito terminam com uma questão aberta que visa reunir contributos sobre aquela que poderá ser a mais relevante consequência que a COVID-19 poderá provocar, em 2020, no tecido cultural português. As respostas obtidas nos dois primeiros meses do estudo foram, como seria de esperar, muito diversificadas. Destaca-se, no entanto, que as preocupações elencadas por organizações e por profissionais do setor cultural foram muito semelhantes.

A grande maioria dos respondentes considera que a COVID-19 terá, no ano de 2020, impactos (muito) negativos no setor cultural português, não só devido ao adiamento/cancelamento de eventos, à suspensão de atividades e ao encerramento de instalações, mas também devido à incerteza do que pode acontecer à medida que as restrições impostas ao setor cultural forem sendo levantadas, mas essencialmente por problemas estruturais do setor cultural, que podem ser agudizados pela pandemia.

\section{NOTAS EM JEITO DE CONCLUSÃO}

O projeto "Impactos da COVID-19 no setor cultural português” só estará concluído em março de 2021, contudo os resultados preliminares do primeiro trimestre indiciam que a contaminação do setor cultural português pela COVID-19 se está a revelar dramática.

Não obstante a considerável cobertura mediática das consequências da COVID-19 no setor cultural português, ficou nítido que a cultura não constituiu uma prioridade editorial, nomeadamente no que concerne às chamadas de primeira página, tendo sido predominantes notícias referentes aos impactos negativos e dado pouco destaque às iniciativas do Ministério da Cultura para apoiar o setor. O tempo de reação dos diversos protagonistas do setor foi muito distinto. O CENA-STE foi uma das primeiras organizações representativas do setor a alertar para os problemas que se avizinhavam, com ou sem COVID-19 e o município do Porto foi uma das primeiras autarquias a reunir com entidades do setor privado para definir estratégias para o turismo e para a cultura. Enquanto as preocupações da maioria das entidades públicas e do ministério se centravam em encerrar espaços e cancelar atividades, as organizações e os 
profissionais do setor cultural privado reagiram de forma muito rápida e espontânea, ainda antes de ser declarada a situação de alerta, promovendo a transição da oferta cultural do espaço físico para o mundo virtual. A adaptação da oferta cultural, associada ao confinamento, voluntário ou obrigatório, foram dois dos motivos que contribuíram para que também tenha havido impactos positivos da COVID-19 no setor cultural português - observou-se, para além de um natural incremento das dinâmicas e oferta e consumos culturais associadas a plataformas online, um aumento histórico das audiências de televisão. Os ecos das iniciativas do Ministério da Cultura para apoiar o setor começaram a surgir na segunda quinzena do mês de março, mas a criação de um website específico não concorreu substantivamente para a proliferação de notícias nos média sobre como o ministério estava a reagir para colmatar grande parte dos problemas que o setor estava a sentir de forma muito particular.

As organizações e os profissionais do setor cultural, auscultados através de um inquérito por questionário, não deixaram margem para dúvidas revelando que, se não forem tomadas medidas urgentes, substantivas e estruturantes, o setor cultural português poderá sofrer danos irreparáveis fruto da pandemia. Os dados revelam que, para além das perdas imediatas que as medidas transversais e setoriais não estão a ser capazes de dar uma resposta cabal, no final de 2020 poderemos assistir, para além de um agudizar da precarização (que já era uma realidade muito presente antes da COVID-19), um incremento no desemprego de profissionais do setor cultural que tinham contratos de trabalho.

Em Portugal, aos constrangimentos provocados pelo inesperado da situação, acrescem as debilidades estruturais que se têm traduzido de múltiplas e variadas formas ao longo dos anos (e.g. desarticulação intersectorial, desarticulação entre os diversos níveis da administração pública, excesso de protagonistas políticos, precarização, subfinanciamento).

Resta, por isso, a esperança de que a pandemia seja uma oportunidade para operar uma mudança de paradigma, promovendo o diálogo consistente e consequente entre os diferentes protagonistas do setor cultural português. Diálogo que só terá a ganhar se entrar em linha de conta com as múltiplas experiências internacionais onde a cultura não é remetida para um papel secundário em tempos de pandemia (e.g. 
Compendium of Cultural Policies $\&$ Trends $^{1}$, Creative Scotland ${ }^{2}$, International Network for Contemporary Performing Arts $^{3}$, KEA European Affairs ${ }^{4}$, On The Move ${ }^{5}$, Organização de Estados Ibero-americanos ${ }^{6}$ ).

\section{REFERÊNCIAS BIBLIOGRÁFICAS}

Cabrita, A. (2020). Ministério da Cultura avança com medidas excecionais. Expresso. Retirado de https:// expresso.pt/coronavirus/2020-03-23-Ministerio-da-Cultura-avanca-com-medidas-excecionais.

Caetano, M. (2020). Os novos desafios dos museus: menos visitantes e menos receita. Diário de Notícias. Retirado de https://www.dn.pt/edicao-do-dia/17-mai-2020/os-novos-desafios-dos-museus-menos-visitantese-menos-receita-12200898.html.

Cardoso, J. (2020). Entrega digital, mais verbas mais cedo: há novas medidas excepcionais do ICA para agilizar apoios. Público. Retirado de https://www.publico.pt/2020/03/26/culturaipsilon/noticia/entrega-digitalverbas-cedo-ha-novas-medidas-excepcionais-ica-agilizar-apoios-1909552.

Cardoso, M. (2020). COVID-19. Câmara do Porto e privados definem estratégias para turismo e cultura. Expresso. Retirado de https://expresso.pt/coronavirus/2020-03-11-COVID-19.-Camara-do-Porto-e-privadosdefinem-estrategias-para-turismo-e-cultura.

Carrapatoso, M. (2020). COVID-19. As 15 medidas de Fernando Medina para enfrentar a crise económica. Expresso. Retirado de https://expresso.pt/coronavirus/2020-03-25-COVID-19.-As-15-medidas-de-FernandoMedina-para-enfrentar-a-crise-economica.

Centro Europeu de Prevenção e Controlo de Doenças. (2020). Pneumonia cases possibly associated with a novel coronavirus in Wuhan, China. Retirado de https://www.ecdc.europa.eu/sites/default/files/documents/ Threat-assessment-Pneumonia-cases-possibly-associated-to-a-novel-coronavirus-in-Wuhan-China.pdf.

Correia, A. (2020). Escritor Luís Sepúlveda infetado com coronavírus após visitar Portugal. Jornal de Noticias. Retirado de https://www.jn.pt/mundo/escritor-luis-sepulveda-infetado-com-coronavirus-aposvisitar-portugal-11873738.html.

Correia, J. (2020). COVID-19. Mapa interativo mostra estabelecimentos encerrados e eventos cancelados pelo país. Expresso. Retirado de https://expresso.pt/coronavirus/2020-03-12-COVID-19.-Mapa-interativomostra-estabelecimentos-encerrados-e-eventos-cancelados-pelo-pais.

1 Ver https://www.culturalpolicies.net/COVID-19/.

2 Ver https://www.creativescotland.com/resources/professional-resources/COVID-19-directory.

3 Ver https://www.ietm.org/en/system/files/publications/performing_arts_in_times_of_the_pandemic_0.pdf.

4 Ver https://keanet.eu/collaborative-map-COVID-19-policy-measures/.

5 Ver http://on-the-move.org/news/article/20675/corona-virus-resources-arts-culture-and-cultural/?f.

6 Ver https://www.oei.es/cultura-COVID-19/iniciativas. 
Costa, R. (2020). NOS Alive já tem datas para 2021.Jornal de Noticias. Retirado de https://www.jn.pt/artes/ nos-alive-ja-tem-datas-para-2021-12215511.html.

Decreto-Lei no 169-B/2019, de 3 de dezembro, República Portuguesa. Retirado de https://dre.pt/application/ conteudo/126869983.

European Creative Business Network. (2020). Urgent Survey: Effects of COVID-19 ("Coronavirus") on the European Cultural and Creative Industries. Retirado de http://ecbnetwork.eu/urgent-survey-effects-ofCOVID-19-coronavirus-on-the-european-culture-and-creative-industries/.

Instituto Nacional de Estatística I.P. (2016). Conta Satélite da Cultura - 2010-2012. Notas metodológicas. Retirado de https://www.ine.pt/xportal/xmain?xpid=INE\&xpgid=ine_docmetodsec2010 cn\&perfilpagenumber $=2 \&$ perfil $=220674570 \&$ contexto $=c$.

Jornal de Notícias. (2020a). Retirado de https://www.jn.pt/edicao-do-dia/2020-03-02.html.

Jornal de Notícias. (2020b). Retirado de https://www.jn.pt/edicao-do-dia/2020-05-26.html.

La Red Española de Teatros, Auditorios, Circuitos y Festivales de Titularidad Pública. (2020). 52 medidas extraordinarias para afrontar las consecuencias del COVID-19. Retirado de https://www.redescena.net/ redaccion/2020/04/52MedidasCOVIDArtesEscenicasMusica.pdf.

Latour, B. (2020). Imaginar gestos que barrem o retorno da produção pré-crise. Retirado de http://www.brunolatour.fr/sites/default/files/downloads/P-202-AOC-03-20-PORTUGAIS.pdf.

Lopes, M. (2020). Ministério da Cultura anuncia medidas de apoio a artistas e profissionais do sector. Público. Retirado de https://www.publico.pt/2020/03/20/culturaipsilon/noticia/ministerio-culturaanuncia-medidas-apoio-artistas-profissionais-sector-1908682.

Lusa (2020a). COVID-19: Direções regionais de Cultura encerram museus e monumentos. Retirado de https://www.lusa.pt/article/hxO4ynFacBhC3fPxb9k2UjMSZM5iuSI1/COVID-19-direçôes-regionais-decultura-encerram-museus-e-monumentos.

Lusa (2020b). COVID-19: Sindicato insta Governo a garantir direitos dos trabalhadores da Cultura. Retirado de https://www.lusa.pt/article/Hg9xOvEPmRVyCCHi89pCXjMSZM5iuSI1/COVID-19-sindicato-instagoverno-a-garantir-direitos-dos-trabalhadores-da-cultura.

Lusa (2020c). COVID-19: Governo cria endereço para responder a agentes culturais sobre apoios. Retirado de https://www.lusa.pt/article/CNaLKY3IyrAlqciwkvzj0DMSZMSiuSI1/COVID-19-governo-cria-endereçopara-responder-a-agentes-culturais-sobre-apoios.

Lusa (2020d). COVID-19: Sociedade de Autores pede apoio urgente ao Governo para os agentes culturais. Retirado de https://www.lusa.pt/article/vecEQkpqm36FhKVCePaxMjMSZM5iuSI1/COVID19-sociedade-de-autores-pede-apoio-urgente-ao-governo-para-os-agentes-culturais.

Lusa (2020e). COVID-19: Plateia considera fundamental linha de emergência do Ministério da Cultura. Retirado de https://www.lusa.pt/article/7OCNvO00nVbvUBthrUbBBjMSZM5iuSI1/COVID-19-plateiaconsidera-fundamental-linha-de-emergência-do-ministério-da-cultura. 
Lusa (2020f). COVID-19: Fundação GDA promove inquérito para avaliar "situação real" vivida nas Artes. Retirado de https://www.lusa.pt/article/ggPx84ZwYr3DUqd3SjN3LzMSZM5iuSI1/COVID-19-fundaçãogda-promove-inquérito-para-avaliar-situação-real-vivida-nas-artes.

Maia, A. (2020). Coronavírus: há dois casos confirmados em Portugal. Público. Retirado de https://www. publico.pt/2020/03/02/sociedade/noticia/coronavirus-ha-dois-infectados-portugal-1905823.

Martins, J. (2020). Como pode a Cultura ajudar... Público. Retirado de https://www.publico.pt/2020/03/23/ culturaipsilon/opiniao/cultura-ajudar-1908897.

Ministério da Cultura do Perú. (2020). Encuesta sobre impactos y potenciales medidas de amortiguamiento en el sector de las artes, industrias culturales y creativas, y museos en el Perú Retirado de https://www.gob.pe/ institucion/cultura/campañas/919-encuesta-sobre-impactos-y-potenciales-medidas-de-amortiguamiento-enel-sector-de-las-artes-industrias-culturales-y-creativas-y-museos-en-el-peru.

Network of European Museum Organisations (2020). Mapping of the short and long term effect of COVID-19 on museums. Retirado de https://www.ne-mo.org/news/article/nemo/mapping-short-and-long-term-effectof-COVID-19-on-museums.html.

Observatório da Economia Criativa da Bahia da Universidade Federal do Recôncavo da Bahia. (2020). Impactos da COVID-19 na Economia Criativa. Retirado de https://ufrb.edu.br/proext/economiacriativa-COVID19/.

Organização Mundial da Saúde. (2020). Pneumonia of unknown cause - China. Disponível em https://www. who.int/csr/don/05-january-2020-pneumonia-of-unkown-cause-china/en/.

Parlamento Europeu. (2020). COVID-19 impact on culture: new funds must reach creative sectors immediately. Retirado de: https://www.europarl.europa.eu/news/en/press-room/20200326IPR75912/ COVID-19-impact-on-culture-new-funds-must-reach-creative-sectors-immediately.

Público (2020a). COVID-19: plataforma onde empresas podem investir em projectos artísticos já está online. Retirado de https://www.publico.pt/2020/03/31/culturaipsilon/noticia/COVID19-plataformaonde-empresas-podem-investir-projectos-artisticos-ja-online-1910294.

Público (2020b). Sindicato pede aos trabalhadores do espectáculo e do audiovisual para avaliarem as suas perdas. Retirado de https://www.publico.pt/2020/03/19/culturaipsilon/noticia/sindicato-pedetrabalhadores-espectaculo-audiovisual-avaliarem-perdas-1908475.

Público. (2020c). Retirado de https://www.publico.pt/jornal?date=20200320\#\&gid=1\&pid=1.

Público. (2020d). Retirado de https://www.publico.pt/jornal?date=20200522\#\&gid=1\&pid=1.

Secretaria da Cultura do Estado do Ceará. (2020). Impacto da Pandemia da Covid-19 na economia da cultura cearense. Retirado de https://www.secult.ce.gov.br/2020/06/29/secult-divulga-estudo-de-impactoda-pandemia-de-covid-19-na-economia-da-cultura-cearense/. 
Soldano, C. \& Nadais, I. (2020). Os 30 milhôes que António Costa anunciou para a cultura "não são uma boa notícia”. Público. Retirado de: https://www.publico.pt/2020/05/22/culturaipsilon/noticia/30-milhoesantonio-costa-anunciou-cultura-nao-sao-boa-noticia-1917779.

UNESCO. (2020). More than 130 Ministers call for support to culture sector in COVID-19 crisis response. Retirado de: https://en.unesco.org/news/more-130-ministers-call-support-culture-sectorCOVID-19-crisis-response.

Xuanxuan, P. (2016). El “Programa Metrópolis Cultural” de Wuhan. Retirado de http://obs.agenda21culture. net/index.php/es/good-practices/el-programa-metropolis-cultural-de-wuhan. 